Artigo / Article

\title{
Heterozigose para hemoglobinopatias em doadores de sangue do Centro de Hemoterapia de Sergipe
}

\author{
Heterozigosity to hemoglobinopathies in blood donors from the Hemotherapy Center in \\ Sergipe, NE-Brazil
}

Wanessa L. P. Vivas $^{1}$

Danilo S. Rebouças ${ }^{2}$

Amaury L. D. Fabbro

Rosana Cipolotti ${ }^{4}$

\begin{abstract}
As hemoglobinopatias, distúrbios geneticamente determinados da hemoglobina $(\mathrm{Hb})$ humana, estão presentes com freqüência elevada em várias partes do mundo, sendo que no Brasil as Hb anormais $S$ e $C$ são as mais prevalentes. Com o objetivo de identificar a presença de portadores saudáveis de genes para hemoglobinopatias entre doadores de sangue do Centro de Hemoterapia do Estado de Sergipe (Hemose), foram analisadas 1.345 amostras de doadores de sangue. Em todas as amostras foram realizados eritrograma automatizado e eletroforese de hemoglobina em acetato de celulose utilizando-se tampão Tris-EDTA-Borato pH 8,6. As amostras que apresentaram hemoglobinas anormais foram submetidas a teste de falcização, teste de solubilidade e Cromatografia Líquida de Alta Performance (HPLC). Foram identificadas 76 amostras com hemoglobinas anormais (5,6\%), das quais 55 (4,1\%) com traço falciforme (Hb AS), 19 (1,4\%) com Hb AC, uma com Hb AD e outra sugestiva de beta-talassemia. Os resultados encontrados demonstram a necessidade de implantação da triagem para hemoglobinopatias entre doadores de sangue, pois desta maneira o receptor de sangue é beneficiado com produto de melhor qualidade, e o doador com a identificação de uma alteração genética que pode vir a se manifestar em seus descendentes. Rev. bras. hematol. hemoter. 2006;28(4):284-287.
\end{abstract}

Palavras-chave: Traço falciforme; doadores de sangue; Hemoglobina S.

\section{Introdução}

Os distúrbios da hemoglobina $(\mathrm{Hb})$ humana ocorrem em freqüência significativa em todos os continentes. Milhões de pessoas carregam genes para hemoglobinas anormais em várias combinações, com conseqüências que variam de quase imperceptíveis a letais. ${ }^{1}$ Apesar da existência de centenas de hemoglobinopatias hereditárias conhecidas, são três as que representam problema de saúde pública no Brasil: as hemoglobinopatias S e C e a talassemia beta, sendo suas respectivas prevalências na população geral 6\%, 1\% e 5\%. Enquanto as duas primeiras, pela sua alta freqüência entre afro-descendentes, apresentam importância nacional, a talassemia beta encontra-se mais freqüentemente entre os descendentes de europeus, especialmente oriundos da região do mar Mediterrâneo, e é mais encontrada nas regiões Sul e Sudeste do Brasil. ${ }^{2}$

Estudos entre doadores de sangue mostram que a prevalência do traço falciforme é maior nos estados da região Nordeste, porém é encontrada em todas as regiões brasilei-

\footnotetext{
${ }^{1}$ Biomédica, mestre em Ciências da Saúde pela Universidade Federal de Sergipe.

${ }^{2}$ Doutorando em Medicina, bolsista PIBIC-CNPq.

${ }^{3}$ Médico, doutor em Medicina (Medicina Social), professor-adjunto do Departamento de Medicina Social da Faculdade de Medicina de Ribeirão Preto-USP.

${ }^{4}$ Médica, doutora em Medicina (Pediatria), professora-adjunta da Universidade Federal de Sergipe.
}

Instituições: Universidade Federal de Sergipe e Hemocentro de Sergipe.

Correspondência: Wanessa Lordêlo Pedreira Vivas

Av. São João Batista, 638, Ed. Mariana, apt ${ }^{0} 302$ - Ponto Novo

49097-000 - Sergipe-AR - Brasil

Tel.: (79) 3231-6075/ (79)3214-1790/ (79) 9135-5405

E-mail: lordelovivas@yahoo.com.br 
ras, e, por isso, a doença falciforme (DF) é considerada uma das doenças genéticas mais importantes no cenário epidemiológico brasileiro. ${ }^{3}$

Sabe-se que a transfusão de hemácias contendo hemoglobina A ( $\mathrm{Hb} \mathrm{A}$ ) e hemoglobina S (Hb S) possui algumas contra-indicações bem específicas, como em transfusão em recém-nascidos (sobretudo em prematuros), em pacientes com anemia falciforme em crises de falcização e em indivíduos com hipóxia intensa. ${ }^{4}$ Desde 2002 existe no Brasil a recomendação para detecção de Hb S em doadores de sangue. A legislação atual ${ }^{5,6}$ torna obrigatória a investigação de outras hemoglobinas anormais no sangue de todos os doadores. As bolsas contendo sangue com Hb S não devem ser descartadas, mas proibida a transfusão destes componentes em pacientes com hemoglobinopatias, recém-nascidos, pacientes com acidose grave e em transfusão intra-uterina. ${ }^{7-23}$

O presente estudo objetivou identificar portadores saudáveis de genes para hemoglobinopatias entre doadores de sangue do Centro de Hemoterapia de Sergipe (Hemose).

\section{Casuística e Métodos}

Sergipe se localiza na região Nordeste do Brasil e possui 1.903.065 habitantes. O Hemose é o único centro de captação de sangue e distribuição de hemoderivados do estado de Sergipe, recebendo cerca de 2.000 doações por mês. Conforme rotina do Hemose, os potenciais doadores com hemoglobina abaixo de $12 \mathrm{mg} / \mathrm{dl}$ não são encaminhados para doação. Foi realizado um estudo transversal de setembro de 2004 a junho de 2005 analisando-se amostras de sangue de 1.345 doadores.

A seleção dos participantes foi aleatória. Durante a coleta da amostra eram anotados o gênero, idade e cor da pele.

O eritrograma automatizado foi realizado em todas as amostras, que em seguida foram lisadas com saponina a $1 \%$ para a realização da eletroforese alcalina em $\mathrm{pH}$ 8,6 com tampão Tris-EDTA-Borato. ${ }^{24}$ As amostras que apresentaram hemoglobinas anormais foram submetidas ao teste de falcização, teste de solubilidade e Cromatografia Líquida de Alta Performance (HPLC).

O projeto deste estudo foi aprovado pelo Comitê de Ética em Pesquisa envolvendo seres humanos da Universidade Federal de Sergipe em 3 de setembro de 2004. Os resultados foram analisados através de distribuição de freqüências, teste exato de Fisher e teste Qui-Quadrado usando software Epi Info (versão 6.04B).

\section{Resultados}

Foram estudadas amostras de sangue de 1.345 doadores, dos quais $85 \%$ eram do gênero masculino. Foram positivas para hemoglobinopatias $76(5,6 \%)$ das amostras, sendo 55 (4,1\%) AS, 19 (1,4\%) AC, 1 (0,1\%) AD e 1 (0,1\%) sugestivo

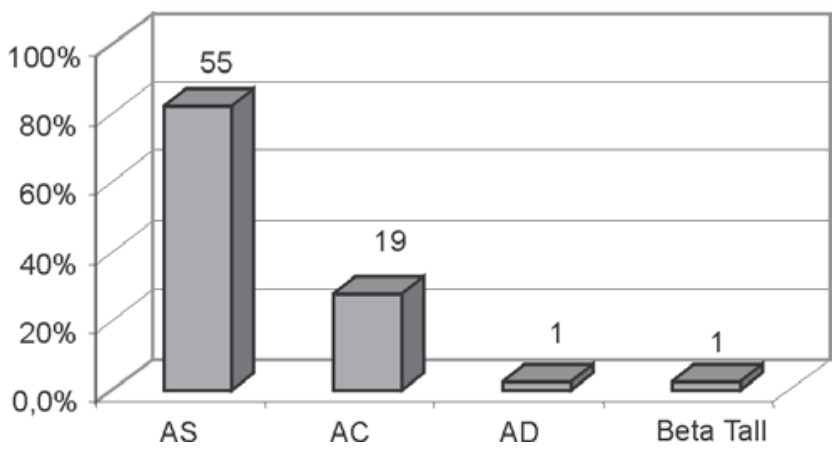

Figura 1. Positividade para hemoglobinopatias entre doadores de sangue

de beta-talassemia (Figura 1). Doadores portadores de $\mathrm{Hb}$ AS correspondem a $73 \%$ e os $\mathrm{Hb}$ AC a $25 \%$ dos portadores de hemoglobinopatias.

Não houve diferença estatisticamente significativa entre as médias de número de hemácias (Hm), dosagem de hemoglobina $(\mathrm{Hb})$, hematócrito (Htc) e hemoglobina corpuscular média (HCM) nos portadores de hemoglobinopatias em relação aos não portadores. Porém, entre as médias de volume corpuscular médio (VCM) e concentração de hemoglobina corpuscular média (CHCM), houve diferença entre os portadores de hemoglobinopatias em relação aos não portadores (Tabela 1).

Foi utilizada a técnica de cromatografia líquida de alta performance (HPLC) para a obtenção dos valores das frações de hemoglobina dos 76 doadores que apresentaram positividade para alguma hemoglobinopatia (Tabela 2).

O teste de solubilidade apresentou-se como um bom discriminador entre doadores normais e com hemoglobinopatias, sendo positivo em $72 \%$ das amostras com hemoglobinopatias e em nenhuma amostra com Hb AA ( $<<0.001)$. O teste de falcização foi positivo em $23 \%$ das amostras com hemoglobinopatias e em nenhuma amostra com $\mathrm{Hb}$ AA $(\mathrm{p}<0.001)$.

\section{Discussão}

O Brasil caracteriza-se por significativa mistura racial, com grande influência na dispersão de genes anormais, notadamente falcemias e talassemias. A distribuição das hemoglobinas normais está relacionada com os grupos raciais que participaram na formação da população de cada região. ${ }^{18}$

Assim, a heterogeneidade étnica da população brasileira, além de variações técnicas, dificulta a comparação dos resultados. No entanto, a Hb S está presente e foi a mais freqüente hemoglobinopatia nesse e em outros estudos. Existe, naturalmente, uma relação com o contingente de africanos que povoaram as diversas regiões. ${ }^{25}$

A presença de hemácias contendo $\mathrm{Hb} \mathrm{S}$ em doadores de sangue brasileiros é representativa, chegando a 2,4\% 
Tabela 1

Distribuição de freqüências dos índices hematimétricos

\begin{tabular}{|c|c|c|c|c|}
\hline & média \pm erro & $\begin{array}{l}\text { desvio } \\
\text { padrão }\end{array}$ & $\begin{array}{c}\text { coeficiente de } \\
\text { variação (\%) }\end{array}$ & $\mathrm{F}$ \\
\hline \multicolumn{5}{|l|}{$\mathrm{Hm}$} \\
\hline $\mathrm{AA}$ & $4.97 \pm 0.05$ & 0.50 & 10.18 & \\
\hline AS & $4.98 \pm 0.07$ & 0.57 & 11.48 & $0.47^{\text {ns }}$ \\
\hline$A C+A D$ & $5.10 \pm 0.10$ & 0.48 & 9.56 & \\
\hline \multicolumn{5}{|l|}{$\mathrm{Hb}$} \\
\hline $\mathrm{AA}$ & $14.34 \pm 0.16$ & 1.42 & 9.93 & \\
\hline AS & $14.02 \pm 0.18$ & 1.39 & 9.94 & $0.87^{\text {ns }}$ \\
\hline$A C+A D$ & $14.19 \pm 0.21$ & 0.95 & 6.75 & \\
\hline \multicolumn{5}{|l|}{$\mathrm{Ht}$} \\
\hline Normais & $43.30 \pm 0.45$ & 3.90 & 9.02 & \\
\hline AS & $41.70 \pm 0.53$ & 3.95 & 9.48 & $2.89^{n s}$ \\
\hline$A C+A D$ & $42.25 \pm 0.65$ & 2.93 & 6.94 & \\
\hline \multicolumn{5}{|l|}{$\mathrm{HCM}$} \\
\hline $\mathrm{AA}$ & $28.79 \pm 0.21$ & 1.87 & 6.52 & \\
\hline AS & $28.27 \pm 0.29$ & 2.15 & 7.63 & $2.03^{\mathrm{ns}}$ \\
\hline$A C+A D$ & $27.90 \pm 0.43$ & 1.94 & 6.98 & \\
\hline \multicolumn{5}{|l|}{ VCM } \\
\hline AA & $86.98 \pm 0.53$ & 4.65 & 5.35 & \\
\hline AS & $84.01 \pm 0.78$ & 5.83 & 6.94 & $8.03^{\star \star \star}$ \\
\hline$A C+A D$ & $82.83 \pm 1.10$ & 4.92 & 5.94 & \\
\hline \multicolumn{5}{|l|}{ CHCM } \\
\hline $\mathrm{AA}$ & $33.10 \pm 0.09$ & 0.81 & 2.45 & \\
\hline AS & $33.44 \pm 0.12$ & 0.94 & 2.84 & $4.82^{\star \star}$ \\
\hline$A C+A D$ & $33.68 \pm 0.12$ & 0.56 & 1.69 & \\
\hline
\end{tabular}

$\mathrm{F}=$ quociente da variância pelo erro; ${ }^{* \star} \mathrm{p}<0.01 ;{ }^{* \star \star} \mathrm{p}<0.001$; ns $=$ não significante

Tabela 2

Valores, média e desvio padrão para as frações de hemoglobinas

\begin{tabular}{cccc}
\hline $\begin{array}{c}\text { Frações de } \\
\text { Hemoglobina }\end{array}$ & Média & $\begin{array}{c}\text { Desvio } \\
\text { Padrão }\end{array}$ & $\begin{array}{c}\text { Amplitude de } \\
\text { variação }\end{array}$ \\
\hline A1 & 59,93 & 05,02 & $53,8-88,3$ \\
A2 & 3,46 & 0,57 & $1,5-7,2$ \\
F & 0,79 & 1,23 & $0,0-4,5$ \\
S & 36,25 & 3,98 & $24,1-41,8$ \\
C & 36,85 & 3,78 & $28,8-43,0$ \\
D & 38,1 & & \\
\hline
\end{tabular}

caracterizando a necessidade potencial da sua detecção laboratorial a nível de triagem. A transfusão destas hemácias pode resultar em efeitos indesejáveis, tanto pela possibilidade de falcização no receptor, como pelas alterações do produto hemoterápico durante o processamento e estocagem. ${ }^{23}$

Os dados obtidos pela eletroforese de hemoglobina, teste de falcização, teste de solubilidade e HPLC indicam que 5,6\% dos doadores de sangue do Centro de Hemoterapia de Sergipe são portadores assintomáticos de hemoglobinopatias, sendo 4,1\% de $\mathrm{Hb}$ AS, $1,4 \%$ de $\mathrm{Hb}$ AC, 0,1\% de $\mathrm{Hb} \mathrm{AD}$ e $0,1 \%$ sugestivo de beta-talassemia. Esses resultados, encontrados entre doadores de sangue em Sergipe são compatíveis aos relatados por Naoum, que mostrou que a prevalência do traço falciforme entre os doadores de sangue é maior nos estados do Nordeste, como a Bahia (5,5\%), ${ }^{26}$ e por Gomes, em Alagoas (2,06\%). ${ }^{27}$

No estudo feito por Melo et al (2000) em 23.981 doadores de sangue de Uberlândia, Minas Gerais, e cidades subjacentes, os autores encontraram 820 (3,42\%) portadores de hemoglobinopatias, onde a $\mathrm{Hb}$ AS foi encontrada em maior prevalência $(2,48 \%$ ) quando comparadas com as outras hemoglobinopatias; o mesmo fato foi encontrado em feito por Tavares e Bernardes ${ }^{22}$ em 483 doadores de sangue do Centro de Hematologia de Sobradinho (Distrito Federal), em que a freqüência de Hb AS foi a predominante (2,96\%), e no Hemonúcleo da Universidade São Francisco, na cidade de Bragança Paulista, São Paulo, onde foi realizado um estudo em 1.846 doadores; desses 1,13\% foi $\mathrm{Hb}$ AS, demonstrando predominância de doadores AS em bancos de sangue do Brasil.. ${ }^{20}$

Nas regiões Sul e Sudeste foi observado 1,9\% de hemoglobinopatias, com predomínio de talassemias ${ }^{28}$ em São Paulo e $11,7 \%$ no Rio Grande do Sul, sendo 9,8\% sugestivos de talassemia. ${ }^{29} \mathrm{~A}$ baixa prevalência de talassemias no presente estudo $(0,1 \%)$ se justifica porque a seleção prévia dos doadores exclui indivíduos anêmicos, além da presumida composição étnica da população.

Neste trabalho verificou-se que se as Hb variantes não puderam ser identificadas apenas pelos métodos eletroforéticos usuais, os quais diferenciaram bem os doadores normais em relação aos que apresentaram algum tipo de hemoglobinopatia. Foi necessária utilização de outras técnicas como o teste de solubilidade e teste de falcização para confirmação principalmente da $\mathrm{Hb} \mathrm{S}$. Evidenciou-se a dificuldade de interpretação das Hb variantes, principalmente daquelas que migraram em posição semelhante à $\mathrm{Hb} \mathrm{S} \mathrm{em} \mathrm{pH}$ alcalino e que poderiam ter sido identificadas incorretamente.

Este estudo indica a necessidade da identificação de hemoglobinas anormais nos doadores de sangue do Hemose, objetivando a melhor qualidade do produto para o receptor e a orientação em saúde para o doador. Sugere-se que amostras sejam triadas através do VCM e da eletroforese de hemoglobina em $\mathrm{pH}$ alcalino, e posteriormente submetidas à HPLC.

\section{Abstract}

Hemoglobinopathies are genetically determined disorders that present in significant high frequencies in certain parts of the world. Despite of the existence of hundreds of known hereditary hemoglobinopathies, Brazilian studies have demonstrated that 
abnormal hemoglobins $S$ and $C$ are the most prevalent. With the objective of identifying the profile of hemoglobinopathies of blood donors at the Hemotherapy Center in the State of Sergipe (Hemose), 1345 samples of blood were analyzed. Initially automatic blood testing and electrophoreses in cellulose acetate using a Tris-EDTABorate buffer at $\mathrm{pH} 8.6$ were carried out for all samples. Samples that presented with abnormal hemoglobins were submitted to testing for the sickle cell trait, solubility test and by High Performance Liquid Chromatography. Seventy-six samples (5.6\%) with abnormal hemoglobins were identified, 55 (4.1\%) of which had the sickle cell trait (Hb AS), 19 (1.4\%) had Hb AC, 1 (0.1\%) had $\mathrm{Hb} A D$ and $1(0.1 \%)$ was suggestive for beta- thalassaemia. The results demonstrate a necessity for the implantation of screening of hemoglobinopathies in blood donors, so that blood recipients benefit from good quality blood and donors from the diagnoses of genetic alterations that may be prevented in descendants. 2006;28(4):284-287.

Key words: Sickle cell trait; blood donor; Hemoglobin S.

\section{Referências Bibliográficas}

1. Thompson MW, Mcinnes RR, Willard HF. Genética Médica 5ed. Rio de Janeiro: Guanabara Koogan,1993.

2. Ramalho AS, Magna LA, Paiva-e-Silva RB. A Portaria $n^{\circ} 822 / 01$ do Ministério da Saúde e as peculiaridades das hemoglobinopatias em saúde pública no Brasil. Cad Saúde Pública, Rio de Janeiro, 2003;19(4):1.195-1.199.

3. Paiva e Silva RB, Ramalho AS, Cassorla ARMS. Anemia falciforme como problema de saúde pública no Brasil. Rev de Saúde Pública, 1993;27:54-58.

4. Prudêncio BCAB, Covas DT, Bonini-Domingos CR. Comparação de metodologia utilizada para a detecção de Hemoglobina S (Hb S) em doadores de sangue. Rev Bras Hematol Hemoter 2000; 22(2):99-109.

5. Brasil. Portaria RDC N. 343. Aprova regulamento técnico para a obtenção, testagem, processamento, e controle de qualidade de sangue e hemocomponentes para uso humano. Diário Oficial da União 2002; 13 dez.

6. Brasil. Portaria RDC N. 153. Aprova regulamento técnico dos Serviços de Hemoterapia. Diário Oficial da União 2004; 14 de jun

7. Marengo-Rowe AJ.Rapid electrophoresis and quantification of haemoglobins on cellulose acetate. J Clin Path 1965;18(6): 790-792.

8. Lepp AC, Bluestein BI. Hemoglobin electrophoresis at alkaline pH on agarose gels. Clinical Chemestry 1978;24(6):936-937.

9. Zago MA, Costa FF, Bottura C. Hemoglobin H disease in three Brazilian families. Revista Brasileira de Genética 1984;7(1):137-147.

10. Zago MA, Paçó-Larson ML. Hemoglobin H disease caused by two gene deletions. Brazilian Journal of Medical and Biologycal Research 1989;22(6):675-681.

11. Ribeiro VS, Araújo JT. Hemoglobin H: laboratory identification. Rev Hosp Clin Fac Med S Paulo 1992;47(4):176-179.

12. Flint J, Harding RM, Boyce AJ, Clegg JB. The population genetics of haemoglobinopathies. Baill Clin Haem 1993;6(1):215-262.

13. Baysal E, Huisman TH. Detection of common alpha-thalassemia2 determinants by PCR. Am J Hematol 1994;46(3):208-213.

14. Molteni S, Frischnecht H, Thormann W. Application of dynamic capillary isoelectric focusing to the analysis of human hemoglobin variants. Electrophoresis 1994;15(1):p.22-30.
15. Foglietta E, Deidda G, Graziani B, Modiano G, Bianco I. Detection of $\alpha$-globin gene disorders by a simple PCR methodology. Haematologica 1996;81:387-396.

16. Mohammad AA, Okorodudu AO, Bissel MG, Dow P, Reger G, Meier A, et al. Clinical application of capillary isoelectric focusing on fused silica capillary for determination of hemoglobin variants. Clinical Chemistry 1997;43(9):1.798-1.799.

17. Guerra-Schinohara EM, Andreguetto AA, Pagliusi RA, D’ Ávila VLB. Importância dos parâmetros: amplitude de variação do tamanho dos eritrócitos (RDW) e dos histogramas de distribuição de volumes celulares no diagnóstico das anemias. NewsLab 1999; 35:118-125.

18. Leoneli GG, Imperial RE, Marchi-Salvador DP, Naoum PC, BoniniDomingos CR. Hemoglobinas anormais e dificuldade diagnóstica. Revista Brasileira de Hematologia e Hemoterapia 2000;22(3): 396-403.

19. Ministério da Saúde (Brasil). Manual de Diagnóstico e Tratamento das Doenças Falciformes. Brasília: Agência Nacional de Vigilância Sanitária, 2002.

20. Acedo MJ, Costa VA, Polimeno NC, Bertuzzo CS. Programa comunitário de hemoglobinopatias: abordagem populacional a partir de doadores de sangue de Bragança Paulista. São Paulo, Brasil. Cad. Saúde Pública São Paulo 2002:(6):18.

21. Melo SMA, Arantes SCF, Botelho FA, Rocha AFS. Prevalência de hemoglobinopatias em doadores de sangue do Hemocentro Regional de Uberlândia MG. Rev Bras Hematol Hemoter 2000;22 Suppl:51.

22. Tavares N, Bernardes R. Hemoglobinas anormais em doadores de sangue do Sobradinho, Distrito Federal. Rev Bras Anal Clin 1980; 12:55-60.

23. Comenalli-Marques JF. Transfusão de hemácias contendo hemoglobina S. Bol Soc Bras Hematol Hemoter 1994;16(166):229-232.

24. Naoum PC, Bonini-Domingos CR. Técnicas laboratoriais para identificação das hemoglobinas normais e anormais In: Naoum PC. (eds.). Hemoglobinopatias e Talassemias. São Paulo: Sarvier, 2001, p. 144-171.

25. Lisot CLA, Silla LMR. Triagem de hemoglobinopatias em doadores de sangue de Caxias do Sul, Rio Grande do Sul, Brasil: prevalência em área de colonização italiana. Cad de Saúde Pública, Rio de Janeiro, 2004,20(6):1.595-1.601.

26. Naoum P. Prevalência e controle da hemoglobina S. Rev. Brás. Hematol Hemoter 2000;22:142-8.

27. Gomes M. Prevalência do traço falcêmico em doadores de sangue do Hemocentro de Alagoas - Hemoal. Rev Laes Haes 2005;115: 147-154.

28. Orlando GM, Noum PC, Siqueira FAM, Bonini-Domingos CR. Diagnóstico laboratorial de hemoglobinopatias em populações diferenciadas. Rev Bras Hematol Hemoter 2000;22:111-121.

29. Lisot CLA. Silla LMR. Triagem de hemoglobinopatias em doadores de sangue de Caxias do Sul, Rio Grande do Sul, Brasil: prevalência em área de colonização italiana. Cad. de Saúde Pública, Rio de Janeiro 2004;20(6):1.595-1.601.

Avaliação: Editor e dois revisores externos

Conflito de interesse: não há.

Recebido: 18/05/2006

Aceito: 27/06/2006 